Alzheimer's disease. Dementia and Geriatric Cognitive Disorders, 37, 315-326.

Coen, R. F. et al. (1997). Distinguishing between patients with depression or very mild Alzheimer's disease using the Delayed-Word-Recall test. Dementia and Geriatric Cognitive Disorders, 8, 244-247.

Dhikav, V. and Anand, K. S. (2012). Caregiver burden of behavioral and psychological symptoms of dementia among Indian patients with Alzheimer's disease. International Psychogeriatrics, 24, 15311532.

Hashidate, H. et al. (2012). Behavioral and psychological symptoms in elderly people with cognitive impairment. Differences between assessment at home and at an adult day-care facility. Nihon Koshu Eisei Zasshi, 59, 532543.

doi:10.1017/\$1041610215001052

\section{Mild cognitive impairment in Parkinson's disease and vascular risk factors among Indian patients}

Mild cognitive impairment (MCI) is often defined as subjective memory complaints with intact activity of daily living without dementia. Its association as a precursor to Alzheimer's disease is well known. However, MCI in Parkinson's disease (PD) is poorly understood. The present small study aimed to measure the frequency of MCI and vascular factors in Indian patients with PD.

MCI in PD is an evolving concept. It is well known that it could be a potential predictor for future cognitive decline and dementia in patients with subsequent Alzheimer's disease. A considerable number of patients with $\mathrm{PD}$ are being diagnosed as PD with MCI (PD-MCI). MCI is common in PD patients without dementia and may be a harbinger of dementia (Hanna-Pladdy et al., 2013).

The Movement Disorder Society Task Force concluded that PD-MCI is common in patients without dementia (mean prevalence, $27 \%$; range, $19 \%-38 \%$ ) and is associated with the subsequent development of Parkinson disease dementia (PDD).

The clinical profile of PD-MCI is heterogeneous, with a range of cognitive domains affected. Overall, non-amnestic, single-domain impairment (i.e. any single non-memory domain) is the most common subtype of PD-MCI (Litvan et al., 2012).

The present study was aimed at assessing the frequency of $\mathrm{MCI}$ and vascular factors (i.e. diabetes and hypertension) in patients with PD. The results were compared to a group of patients diagnosed with MCI in the absence of PD.
Petersen, R. C. (2009). Early diagnosis of Alzheimer's disease: is MCI too late? Current Alzheimer Research, 6, 324-330.

Serra, L. et al. (2010). Are the behavioral symptoms of Alzheimer's disease directly associated with neurodegeneration? Fournal of Alzheimer's Disease, 21, 627-639.

Vikas Dhikav, Mansi SeTHi, Pinki Mishra AND KuljeEt Singh ANAND

Memory Clinic, Department of Neurology, Postgraduate Institute of Medical Education \& Research (PGIMER) \& Dr. Ram Manohar Lohia Hospital, GGS-IP University, New Delhi, 11001, India

Email: vikasdhikav@hotmail.com.

This study was conducted at Post Graduate Institute of Medical Sciences and Research and Dr. RML Hospital, a Tertiary Care center in New Delhi, India. A total of 50 patients were selected randomly from patients presenting to the Department of Neurology and reporting to the Movement Disorder Clinic with a diagnosis of PD and evaluated with regard to subjective memory complaints. Evaluation of MCI was done using the Petersen's criteria for MCI (Petersen, 2009). Also a separate comparison group $(n=26)$, presenting to the Memory Clinic with the diagnosis of MCI was enrolled and patients were incorporated randomly in this group as well.

A total of 14 patients out of $50(28 \%)$ with PD met Petersen's criteria for MCI. Mean age of patients with PD with MCI was $67.34 \pm 9.74$ years and $71.19 \pm 8.14$ years for the comparison group with MCI alone. There was no statistically significant difference between these groups with regard to their mean ages ( $p$ value $>0.05$ ).

Hoehn-Yahr staging was performed for patients with PD $(n=46)$. The Mean Staging Score of those with MCI-PD $(n=12)$ was $2.54 \pm 0.96$ and of those with PD without MCI was $1.62 \pm 1.01$ $(n=34)$ and paired $t$-test showed that both groups differed significantly ( $p$ value $=<0.05$ ).

Prevalence of vascular risk factors i.e. diabetes and hypertension in both groups, i.e. PD-MCI $(n=14)$ and MCI alone $(n=26)$ was made. In the comparison group with MCI, there were a total of 6 patients with diabetes and 16 patients with hypertension out of a total sample size of 26 . In the group of patients with PD, out of a total of 14 patients with PD-MCI, 7 had hypertension and 3 had diabetes. An attempt was made to know if the frequency of diabetes and hypertension differed in patients with MCI and those with PD and MCI. 
There was no significant difference between both groups in regards to diabetes and hypertension $\left(\chi^{2}\right.$ test; $p$ value $>0.05$ ).

In patients with PD and MCI, the frequency of hypertension and diabetes was compared to those without MCI using Fisher's exact test. There was no statistical significant difference between these two groups $(p$ value $=0.3$ )

\section{Discussion}

In the present study, frequency of MCI in PD patients was $28 \%$, which is consistent with the findings from Litvan et al. Another study found that as per Movement Disorder Society criteria involving 109 patients, 28 (26\%) patients had MCI (Melzer et al., 2013).

The mean age of patients with PD-MCI and those in the comparison group was not significantly different. Frequency of hypertension and diabetes in patients with PD-MCI and those with only MCI was not significantly different. This may indicate that vascular factors might be playing an important role in both groups.

A statistically significant difference in means of Hoehn-Yahr staging was found in patients with or without MCI in the Parkinson's group implying that the presence of MCI might indicate greater disease severity.

The impact of PD dementia is substantial and has major functional and socioeconomic consequences and could be a crucial step in identifying future effective interventions (Hanna-Pladdy et al., 2013).

The underlying causes of MCI in PD are unclear. MCI and dementia contribute to poor quality of life among patients with PD (Kandiah, 2013). Vascular factors have received attention in PDMCI. Logistic regression analyses demonstrated white matter hyperintensities (WMH) on Magnetic Resonance Imaging of the brain, to be associated with PD-MCI independent of age, education, and vascular risk factors. Increasing WMH volume was associated with lower performance on executive function, memory, and language (Kandiah, 2013). While there was no significant difference in the frequency of vascular factors between both groups, this might have been due to small sample size and vascular factors might contribute to the presence of MCI in patients with PD. Larger studies would be valuable to investigate this further.

Kandiah has reported that cognitive decline was associated with worse motor and non-motor features, suggesting that this reflects a faster progressive phenotype. This is consistent with the staging score differences found in our study.

\section{Conflict of interest}

None.

\section{References}

Hanna-Pladdy, B., Jones, K., Cabanban, R., Pahwa, R. and Lyons, K. E. (2013). Predictors of mild cognitive impairment in early-stage Parkinson's disease. Dementia and Geriatrics Cognitive Disorders Extra, 3, 168178.

Kandiah, N. (2013). Cerebral white matter hyperintensity in Parkinson's disease: a major risk factor for mild cognitive impairment. Parkinsonism and Related Disorders, 19, 680-683.

Litvan, I. et al. (2012). Diagnostic criteria for mild cognitive impairment in Parkinson's disease: movement disorder society task force guidelines. Movement Disorders, 27, 349-356.

Melzer, T. R. et al. (2013). White matter microstructure deteriorates across cognitive stages in Parkinson disease. Neurology, 80, 1841-1849.

Petersen, R. C. (2009). Early diagnosis of Alzheimer's disease: is MCI too late? Current Alzheimer Research, 6, 324-330.

\section{VIKAS DHIKaV, MANSI SETHI AND KuljeEt Singh ANAND}

Memory Clinic, Department of Neurology, Postgraduate Institute of Medical Education \& Research (PGIMER), GGS-IP University, New Delhi, 110001, India

Email: vikasdhikav@hotmail.com 